\title{
VARIABILIDADE ESPACIAL DE ATRIBUTOS MINERALÓGICOS DE UM LATOSSOLO SOB DIFERENTES FORMAS DO RELEVO. I - MINERALOGIA DA FRAÇÃO ARGILA ${ }^{(1)}$
}

\author{
Livia Arantes Camargo ${ }^{(2)}$, José Marques Júnior ${ }^{(3)}$, Gener Tadeu \\ Pereira $^{(4)}$ \& Renata Alves Horvat ${ }^{(5)}$
}

\begin{abstract}
RESUMO
A distribuição dos minerais da fração argila depende de condições pedoambientais específicas. $O$ estudo das relações entre atributos desses minerais e locais específicos de sua ocorrência na paisagem torna-se importante para o entendimento das relações entre a mineralogia e os demais atributos do solo. Este trabalho teve como objetivo avaliar os atributos cristalográficos, as razões Gt/ $(\mathrm{Gt}+\mathrm{Hm})$ e $\mathrm{Ct} /(\mathrm{Ct}+\mathrm{Gb})$ dos minerais da fração argila (hematita, goethita, caulinita e gibbsita) e o padrão de distribuição espacial destes em um Latossolo Vermelho eutroférrico sob cultivo de cana-de-açúcar há mais de 30 anos, com colheita no sistema cana crua. As amostras de solo foram coletadas nos pontos de cruzamento de uma malha, georreferenciados, com intervalos regulares de $10 \mathrm{~m}$, e dimensão de $100 \times 100 \mathrm{~m}$, na profundidade de $0,2-0,4 \mathrm{~m}$, totalizando 119 pontos, em uma área de 1 ha. As amostras de solos foram analisadas quimicamente. Posteriormente, a fração argila foi submetida à difração de raios $\mathrm{X}$. Foram feitas as análises estatísticas e geoestatísticas dos atributos cristalográficos dos minerais analisados. Mapas de krigagem foram realizados para a visualização da variabilidade espacial dos atributos. Todos os atributos estudados apresentaram dependência espacial, e os atributos mineralógicos apresentaram-se relacionados com variações das formas do relevo. A gibbsita apresentou o maior diâmetro médio do cristal (DMC) entre os minerais da fração argila estudados, e a goethita foi o mineral que apresentou a maior variação do DMC e da largura à meia altura. Os maiores valores do DMC da
\end{abstract}

\footnotetext{
(1) Recebido para publicação em dezembro de 2007 e aprovado em setembro de 2008.

(2) Mestranda do Programa de Pós-Graduação em Agronomia (Produção Vegetal) da FCAV/UNESP, Campus de Jaboticabal. Via de acesso Prof. Paulo Donato Castellani s/n ${ }^{\circ}$, CEP 14870-900, Jaboticabal (SP). Bolsista MSc. FAPESP. E-mail: li_arantes@yahoo.com.br

${ }^{(3)}$ Professor Dr. do Departamento de Solos e Adubos, FCAV/UNESP. E-mail: marques@fcav.unesp.br

(4) Professor Dr. do Departamento de Ciências Exatas, FCAV/UNESP. E-mail: genertp@fcav.unesp.br

${ }^{(5)}$ Aluna de graduação em Agronomia, Departamento de Solos e Adubos, FCAV/UNESP. Bolsista IC FAPESP. E-mail: renata_horvat@yahoo.com.br
} 
goethita, hematita e gibbsita foram encontrados no compartimento II, caracterizado por uma área côncavo-convexa.

Termos de indexação: hematita, goethita, caulinita, gibbsita, geoestatística, krigagem.

\title{
SUMMARY: SPATIAL VARIABILITY OF MINERALOGICAL ATTRIBUTES OF AN OXISOL UNDER DIFFERENT RELIEF FORMS. I-CLAY FRACTIONMINERALOGY
}

\begin{abstract}
The distribution of the clay fraction minerals depends on specific soil-environmental conditions. The study of the relationship between properties of these minerals and specific sites of landscape occurrence is important to understand the relationship between the mineralogy and other soil attributes. This study aimed to evaluate the degree of cristallinity of goethite, hematite, kaolinite and gibbsite and the spatial pattern in an Oxisol under sugar cane for 30 years. In a grid with regular $10 \mathrm{~m}$-intervals, 119 samples were collected at a depth of 0.2-0.4 m. The crystallographic attributes of the minerals were analyzed statistically and geostatistically. Kriging maps were drawn to visualize the spatial variability of attributes. All attributes were spatially dependent, and the mineralogical attributes were related to variations in the relief forms. The largest mean crystal diameter (MCD) among the studied clay minerals was observed for gibbsite, and the greatest variation in the MCD and full width at half maximum in the mineral goethite. The highest values of the MCD of goethite, hematite and gibbsite were found in the compartment II characterized by a concave-convex area.
\end{abstract}

Index terms: hematite, goethite, kaolinite, gibbsite, geostatistics, kriging.

\section{INTRODUÇÃO}

Além do teor, os atributos cristalográficos dos minerais da fração argila são fortemente dependentes das características pedoambientais (Curi \& Franzmeier, 1984). Caulinita, gibbsita, hematita e goethita constituem os principais minerais da fração argila dos Latossolos brasileiros e muito interferem no comportamento dos atributos físicos e químicos dos solos, justificando os estudos que objetivam sua caracterização espacial (Strauss et al., 1997; Ferreira et al., 1999a,b; Pedrotti et al., 2003).

Schwertmann \& Taylor (1989) citam os diferentes óxidos de ferro que podem ocorrer no solo e suas características, afirmando que goethita e hematita são os minerais de maior ocorrência em solos intemperizados das regiões tropicais e subtropicais e que, geralmente, ocorrem associados. Segundo Kämpf \& Curi (2000), os óxidos e hidróxidos de ferro e alumínio são importantes indicadores pedogenéticos por terem sua formação influenciada pelas condições do ambiente e por persistirem por longo tempo no solo.

A complexidade do ambiente em que são formados os minerais da fração argila explica a ocorrência de populações com características distintas desses minerais. Schwertmann \& Kämpf (1985) encontraram diferenças entre características de goethitas e hematitas entre solos do sul e do centro do País. Inda Júnior \& Kämpf (2005), estudando o comportamento de populações de goethita e hematita em Latossolos, concluí- ram que as goethitas se mostraram mais heterogêneas quanto à substituição por $\mathrm{Al}^{3+}$, morfologia $\mathrm{e}$ cristalinidade; já a população de hematita apresentou-se mais homogênea. Populações de caulinita e gibbsita também apresentam variações em seus atributos cristalográficos em pedoambientes específicos (Pedrotti et al., 2003).

Sabendo-se que a mineralogia dos solos é resultante da integração dos processos e fatores de formação, muitos autores têm estudado o relevo como fator condicionador de teores e cristalinidade dos minerais da fração argila. Curi \& Franzmeier (1984), estudando uma toposseqüência de Latossolos desenvolvidos de basaltos no Planalto Central (Goiás), encontraram a maior concentração de gibbsita na posição mais elevada de uma toposseqüência e maior concentração de caulinita nas posições mais baixas, associadas a goethitas de menor diâmetro médio do cristal.

Apoiando-se na idéia de formação poligenética dos solos de ambientes tropicais (Muggler, 1998), Inda Júnior \& Kämpf (2005) alertam sobre os procedimentos de análises tradicionais (ex.: difratometria de raios $\mathrm{X}$ ) que refletem somente valores médios da população, os quais podem ser conflitantes com as condições ambientais atuais (Motta \& Kämpf, 1992; Muggler, 1998), estando em aparente desacordo com a abordagem da formação dos óxidos de ferro pedogênicos.

Análises estatísticas tradicionais, baseadas na independência das observações, não são adequadas para estudos de variabilidade espacial de atributos do solo 
e devem ser complementadas por análises espaciais que consideram as correlações entre observações vizinhas e maior amostragem da população (Hamlett et al., 1986). A análise geoestatística permite detectar a variabilidade e distribuição espacial dos atributos estudados e, portanto, constitui importante ferramenta na análise e descrição detalhada da variabilidade dos atributos do solo (Vieira, 2000; Carvalho et al., 2002; Vieira et al., 2002).

Os Modelos Digitais de Elevação (MDE) são ferramentas que, quando associadas aos mapas de variabilidade resultantes da análise geoestatística, permitem a visualização e o entendimento da distribuição espacial dos atributos do solo (Hammer et al., 1995). Além disso, com o uso desses modelos, podem-se estabelecer relações das influências da topografia, da hidrologia, das classes de declividade do terreno e da quantidade e do tipo de vegetação sobre as classes de solos e, com isso, aperfeiçoar essas informações em levantamentos de solos, estabelecendo limites mais exatos entre as classes (Klingebiel et al., 1987).

Nesse sentido, a espacialização dos teores dos minerais da fração argila, levando em consideração as formas do relevo, constitui estudo básico para auxiliar no entendimento da relação entre esses minerais e a variação dos atributos do solo, tornando possível a transferência desses conhecimentos para áreas semelhantes.

O objetivo deste trabalho foi caracterizar a variabilidade espacial dos atributos mineralógicos dos minerais da fração argila de um Latossolo sob cultivo de cana-deaçúcar, em áreas com variações das formas do relevo.

\section{MATERIAL E MÉTODOS}

\section{Localização, caracterização do meio físico e critério de amostragem}

A área de estudo localiza-se no nordeste do Estado de São Paulo, no município de Guariba (SP). O clima da região, segundo a classificação de Köppen, é do tipo mesotérmico com inverno seco (Cwa), com precipitação média de $1.400 \mathrm{~mm}$, com chuvas concentradas no período de novembro a fevereiro. A vegetação natural é constituída por floresta tropical subcaducifólia. O solo foi classificado como Latossolo Vermelho eutroférrico textura muito argilosa (LVef), seguindo critérios da Embrapa (2006). A área possui histórico de 30 anos de cultivo de cana-de-açúcar, sendo 16 anos com colheita sob sistema cana crua. A caracterização dos minerais da fração argila caulinita $(\mathrm{Ct})$, gibbsita, $(\mathrm{Gb})$, hematita $(\mathrm{Hm})$ e goethita $(\mathrm{Gt})$ foi realizada em amostras de solo coletadas no cruzamento de pontos georreferenciados de uma malha, com intervalos regulares de $10 \mathrm{~m}$, na profundidade de $0,2-0,4 \mathrm{~m}$, com dimensão de 100 x 100 m, totalizando 119 pontos em uma área de 1 ha.

Intensas investigações de campo e auxílio do Modelo Digital de Elevação do terreno possibilitaram o mapeamento de dois compartimentos na área (I e II) (Figura 1).

O compartimento I é caracterizado pela forma linear com declive menos acentuado, e o compartimento II, por uma área com forma côncavoconvexa (Troeh, 1965), e a diferença entre os valores máximo e mínimo da altitude na área total é de $5 \mathrm{~m}$.

\section{Métodos de laboratório}

As amostras de solo foram tratadas com $\mathrm{NaOH}$ $0,5 \mathrm{~mol} \mathrm{~L}^{-1} \mathrm{e}$ submetidas à agitação mecânica por 10 min para a dispersão das partículas. Após esse tratamento, a fração areia foi retirada em peneira de $0,05 \mathrm{~mm}$. A separação da fração silte ocorreu por centrifugação (1.600 rpm) e o tempo da operação foi determinado pela temperatura das amostras no momento da análise. A suspensão de argila foi floculada com $\mathrm{HCl}$ concentrado e centrifugada (2.000 rpm, por $2 \mathrm{~min})$. Posteriormente, realizou-se a eliminação dos óxidos de ferro da fração argila, utilizando o método ditionito-citrato-bicarbonato (DCB), segundo Mehra \& Jackson (1960). A análise da fração argila desferrificada foi realizada por meio da difratometria de raios $\mathrm{X}$ (DRX).

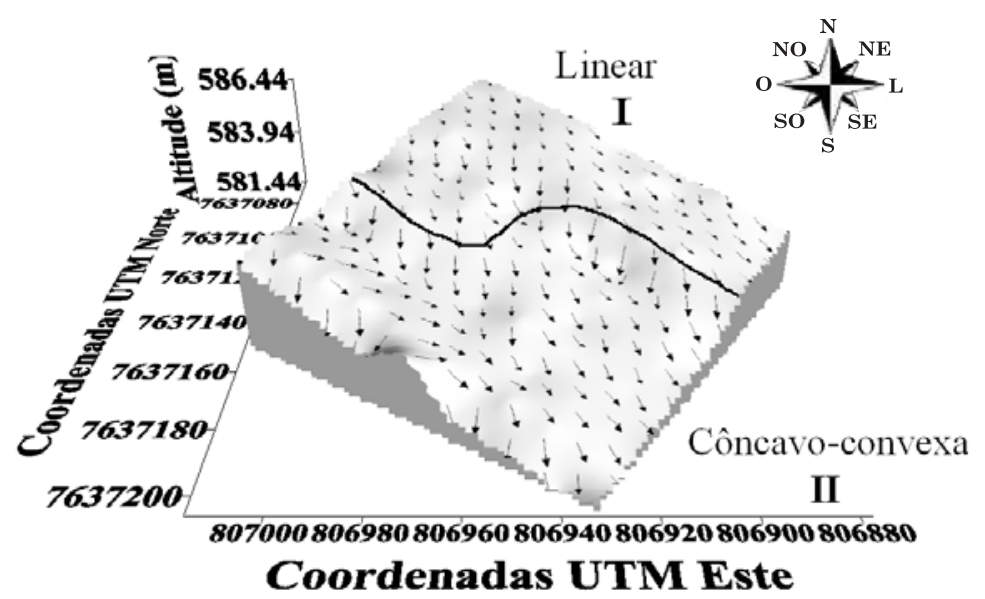

Figura 1. Modelo Digital de Elevação da área de estudo. O sentido e tamanho das setas indicam a modelagem do declive do terreno e, conseqüentemente, o fluxo de água. 
A caracterização da goethita e da hematita por meio da DRX foi feita após tratamento da fração argila com $\mathrm{NaOH} 5 \mathrm{~mol} \mathrm{~L}^{-1}$ (100 mL solução/1 g argila), para verificar a concentração dos mesmos, segundo método de Norrish \& Taylor (1961), modificado por Kämpf \& Schwertmann (1982). Foram adicionados $10 \% \mathrm{em}$ peso de sílica-gel moída para a manutenção de concentração mínima de ácido silícico na solução de $\mathrm{NaOH}$ $5 \mathrm{~mol} \mathrm{~L}^{-1}$, evitando mudanças na substituição em $\mathrm{Al}$ e cristalinidade da goethita (Kämpf \& Schwertmann, 1982).

Para evitar que a leitura dos difratogramas fosse dificultada pela sodalita, as amostras foram lavadas com solução de $\mathrm{HCl} 0,5 \mathrm{~mol} \mathrm{~L}^{-1}(100 \mathrm{ml}$ de solução/ $1 \mathrm{~g}$ de argila), em agitação por $4 \mathrm{~h}$. Para corrigir os desvios no posicionamento (d) dos reflexos estudados, foram acrescentados às amostras $10 \% \mathrm{em}$ peso de cloreto de sódio moído e peneirado em malha de $0,10 \mathrm{~mm}$, antes de serem difratados. A difração de raios $\mathrm{X}$ foi realizada com as amostras preparadas pelo método do pó, em aparelho HGZ equipado com cátodo de Co e filtro de Fe e radiação $\mathrm{K} \alpha(20 \mathrm{~mA}, 30 \mathrm{kV})$ para a difração da $\mathrm{Hm}$ e Gt, e cátodo de $\mathrm{Cu}$ com filtro de $\mathrm{Ni}$ para a difração da $\mathrm{Ct}$ e $\mathrm{Gb}$. A velocidade de varredura empregada foi de $1^{\circ} 2 \theta /$ min e amplitude de 23 a $49^{\circ}$ para caracterização da Hm e Gt e de 11 a $19^{\circ}$ para caracterização da $\mathrm{Ct}$ e $\mathrm{Gb}$.

Foram utilizados para avaliação os reflexos a $\mathrm{Ct}$ (001), Gb (002), Hm (012 e 110), Gt (110 e 111). O diâmetro médio do cristal (DMC) da $\mathrm{Ct}$ e $\mathrm{Gb}$ foi calculado a partir da largura à meia altura (LMA) e da posição dos reflexos dos minerais $\mathrm{Ct}$ (001) e Gb (002). O DMC da Hm e Gt foi calculado a partir da LMA e da posição dos reflexos dos minerais $\mathrm{Hm}$ (110) e Gt (110). Foi utilizada a equação de Scherrer (Schulze, 1984) para o cálculo do DMC. A razão goethita/(goethita + hematita) - [Gt/(Gt + Hm)] foi calculada empregando-se as áreas dos reflexos $\mathrm{Hm}$ (012) e Gt (110). Para o cálculo da razão Gt/(Gt + $\mathrm{Hm}$ ), a área do reflexo Gt (110) foi multiplicada por 0,35 devido à intensidade de $35 \%$ da $\mathrm{Hm}$ (012) (Kämpf \& Schwertmann, 1998). A razão caulinita/(caulinita + gibbsita $)-[\mathrm{Ct} /(\mathrm{Ct}+\mathrm{Gb})]$ foi calculada empregando- se as áreas dos reflexos Ct (001) e Gb (002). O Fe ditionito $\left(\mathrm{Fe}_{\mathrm{d}}\right)$ foi transformado em teor de goethita $\mathrm{e}$ hematita (Dick, 1986).

\section{Análise estatística e geoestatística}

Os dados foram submetidos à análise estatística descritiva, calculando-se a média, a mediana, a variância, o desvio-padrão, a assimetria, a curtose, o máximo, o mínimo, o coeficiente de variação e o tipo de distribuição. Na análise estatística dos dados, foi utilizado o programa Minitab (2000).

Para caracterização do padrão da variabilidade espacial, utilizou-se a análise geoestatística (Vieira, 2000). Foram construídos semivariogramas com base no cálculo da semivariância $\gamma(\mathrm{h})$ por meio da seguinte equação:

$$
\hat{\gamma}(h)=\frac{1}{2 N(h)} \sum_{i=1}^{N(h)}\left[Z\left(x_{i}\right)-Z\left(x_{i}+h\right)\right]^{2}
$$

em que: $\hat{\gamma}(h)$ é a semivariância estimada a partir dos dados experimentais; Z são os valores medidos nos pontos xi e xi $+\mathrm{h}$; $\mathrm{N}$ (h) é o número de pares de valores medidos separados por uma distância h. Os modelos foram ajustados por meio do programa GS+ (Robertson, 1998). Em caso de dúvida entre mais de um modelo para o mesmo semivariograma, foi utilizada a técnica de validação cruzada. Observada a dependência espacial entre as amostras definida pelo semivariograma, é possível estimar valores em que a variável não foi medida, fazendo uso da krigagem, segundo Vieira et al. (2002). A confecção dos mapas de krigagem foi realizada utilizando-se o programa Surfer (1999).

\section{RESULTADOS E DISCUSSÃO}

Os resultados da avaliação estatística dos atributos cristalográficos e das razões $\mathrm{Gt} /(\mathrm{Gt}+\mathrm{Hm})$ e $\mathrm{Ct} /(\mathrm{Ct}+\mathrm{Gb})$ estudados na área total podem ser observados no quadro 1. Os valores das razões $\mathrm{Gt} /(\mathrm{Gt}+\mathrm{Hm})$ e $\mathrm{Ct} /$ $(\mathrm{Ct}+\mathrm{Gb})$ apresentaram distribuição normal. A

Quadro 1. Estatísticas descritivas dos atributos cristalográficos da hematita (Hm), goethita (Gt), caulinita (Ct) e gibbsita (Gb), e razão $\mathrm{Gt} /(\mathrm{Gt}+\mathrm{Hm})$ e $\mathrm{Ct} /(\mathrm{Ct}+\mathrm{Gb})$ de 119 amostras coletadas na profundidade de 0,2-0,4 m

\begin{tabular}{|c|c|c|c|c|c|c|c|c|}
\hline & & Média & Variância & Assimetria & Curtose & Desvio-padrão & $\mathrm{CV}^{(3)}$ & $p^{(4)}$ \\
\hline & & & & & & & $\%$ & \\
\hline \multirow{4}{*}{$\mathrm{LMA}^{(1)}$} & $\mathrm{Hm}$ & 0,34 & 0,001 & 0,98 & 1,02 & 0,03 & 10 & 0,005 \\
\hline & Gt & 0,33 & 0,023 & $-0,29$ & $-0,35$ & 0,15 & 47 & 0,005 \\
\hline & $\mathrm{Ct}$ & 0,44 & 0,004 & 0,14 & 1,20 & 0,06 & 14 & 0,005 \\
\hline & $\mathrm{Gb}$ & 0,28 & 0,000 & 0,50 & $-1,32$ & 0,01 & 4 & 0,005 \\
\hline \multirow[t]{4}{*}{$\mathrm{DMC}^{(2)}$} & $\mathrm{Hm}$ & 71,16 & 209,524 & $-0,18$ & $-1,08$ & 14,47 & 20 & 0,005 \\
\hline & $\mathrm{Gt}$ & 63,20 & 543,940 & $-0,30$ & $-0,15$ & 23,32 & 37 & 0,005 \\
\hline & $\mathrm{Ct}$ & 34,36 & 118,220 & 2,14 & 5,35 & 10,87 & 32 & 0,005 \\
\hline & $\mathrm{Gb}$ & 90,60 & 132,88 & $-0,34$ & $-0,53$ & 11,53 & 13 & 0,005 \\
\hline \multicolumn{2}{|c|}{$\mathrm{Gt} /(\mathrm{Gt}+\mathrm{Hm})$} & 0,11 & 0,010 & 0,46 & $-0,31$ & 0,06 & 52 & 0,150 \\
\hline \multicolumn{2}{|c|}{$\mathrm{Ct} /(\mathrm{Ct}+\mathrm{Gb})$} & 0,38 & 0,0038 & 0,37 & 0,54 & 0,06 & 16 & 0,601 \\
\hline
\end{tabular}


normalidade dos dados não é uma exigência da geoestatística, sendo conveniente apenas que a distribuição não seja muito assimétrica, o que poderia comprometer as análises (Cressie, 1991). Neste estudo, todos os atributos, exceto o DMC da Ct e a LMA da $\mathrm{Hm}$, apresentaram distribuição simétrica indicada pelos valores de assimetria próximos de zero (Quadro 1).

O mineral que apresenta o maior grau de cristalinidade é a Gb, com valor médio de DMC igual a 90,60 nm e LMA de $0,28^{\circ} 2 \theta$. O menor foi a Ct com o DMC igual a $34,36 \mathrm{~nm}$ e LMA de $0,44^{\circ} 2 \theta$. Os valores médios da LMA e DMC da Gb estão de acordo com os estudos realizados por Ghidin et al. (2006) em um LatossoloVermelho distroférrico (LVdf) do Estado do Paraná. Esses autores relatam o maior grau de cristalinidade da Gb em relação aos óxidos de Fe e a predominância deste mineral em horizontes do LVdf. Resultados semelhantes também foram encontrados por Melo et al. (2001). A predominância da Gb na profundidade estudada pode ser resultante da remoção do Si para horizontes inferiores, segundo Curi \& Franzmeier (1984).

Segundo Fitspatrick \& Schwertmann (1982), o maior grau de cristalinidade é evidenciado pelo maior DMC e menor LMA. Schwertmann \& Carlson (1994) relatam que a variação do DMC nos óxidos de Fe é função das diferenças em seus ambientes de formação, taxa de formação dos óxidos de Fe, inibidores de cristalização, como matéria orgânica e idade do mineral. Kämpf \& Curi (2000) relatam que a formação dos óxidos de Fe requer condições específicas, como temperatura, umidade, $\mathrm{pH}$ e matéria orgânica.

Analisando os valores do CV (Quadro 1), observase que houve maior variabilidade para os atributos cristalográficos da Gt. Inda Júnior \& Kämpf (2005), comparando os mesmos atributos, verificaram os maiores valores de $\mathrm{CV}$ para Gt em relação à $\mathrm{Hm}$. Esses autores relatam que a maior homogeneidade das populações de hematita está relacionada com a maior especificidade de sua formação, sendo menos sensíveis às mudanças do meio, ao contrário da goethita.

Comparando os valores dos atributos nos compartimentos I e II (Quadro 2), observa-se o comportamento diferenciado significativo da LMA e DMC da $\mathrm{Hm}$ e Gt e da razão Gt/(Gt+Hm). Esses valores revelam a influência das formas do relevo no comportamento dos atributos.

Analisando esses valores, observa-se a necessidade da análise de dependência espacial nos compartimentos, cujos resultados estão apresentados no quadro 3 e na figura 2 .

Todos os atributos estudados apresentaram dependência espacial, expressa por meio dos ajustes dos modelos de semivariogramas (Figura 2) (Vieira, 1995, 2000). O modelo de melhor ajuste aos semivariogramas dos atributos LMA (Hm, Gt, Ct e $\mathrm{Gb}), \mathrm{DMC}(\mathrm{Ct}$ e $\mathrm{Gb})$, relação $\mathrm{Gt} /(\mathrm{Gt}+\mathrm{Hm})$ e $\mathrm{Ct} /(\mathrm{Ct}+\mathrm{Gb})$ foi o exponencial, enquanto o modelo esférico se ajustou aos semivariogramas do DMC da $\mathrm{Hm}$ e Gt. Para vários autores (Vieira, 1997; Souza et al., 2001; Carvalho et al., 2002), o modelo esférico é predominante nos trabalhos de ciência do solo. Mcbratney \& Webster (1986) citaram os modelos esférico e exponencial como os mais encontrados.

$\mathrm{O}$ efeito pepita $\left(\mathrm{C}_{0}\right)$ (Quadro 3) representa a variabilidade não explicada, considerando a distância de amostragem utilizada (Vieira, 2000). Neste estudo, o DMC da Hm, da Gt e da Gb apresentou valores elevados de efeito pepita em relação aos demais atributos, indicando um erro maior nas estimativas desses atributos.

A relação entre o efeito pepita $\left(\mathrm{C}_{0}\right)$ e o patamar do semivariograma $\left(\mathrm{C}_{0}+\mathrm{C}_{1}\right)$ indica o grau da dependência espacial das variáveis em estudo (Trangmar et al., 1985). O atributo LMA da $\mathrm{Hm}$ e da Ct e o DMC da Ct apresentaram dependência espacial forte $\left[\mathrm{C}_{0} /\left(\mathrm{C}_{0}+\mathrm{C}_{1}\right)\right.$ $\leq 25 \%$, enquanto os demais atributos apresentaram

Quadro 2. Valores médios dos atributos mineralógicos nos compartimentos I e II

\begin{tabular}{|c|c|c|c|c|}
\hline & & $\begin{array}{c}\text { Compartimento I } \\
\text { Média }\end{array}$ & $\begin{array}{c}\text { Compartimento II } \\
\text { Média }\end{array}$ & Teste t \\
\hline \multirow[t]{4}{*}{$\mathrm{LMA}^{(1)}$} & $\mathrm{Hm}$ & $0,342 \mathrm{a}$ & $0,333 \mathrm{~b}$ & 2,11 * \\
\hline & $\mathrm{Gt}$ & $0,362 \mathrm{a}$ & $0,298 \mathrm{~b}$ & 2,22 * \\
\hline & $\mathrm{Ct}$ & $0,443 \mathrm{a}$ & $0,445 \mathrm{a}$ & $-0,21 \mathrm{~ns}$ \\
\hline & $\mathrm{Gb}$ & $0,283 \mathrm{a}$ & $0,279 \mathrm{a}$ & $1,68 \mathrm{~ns}$ \\
\hline \multirow[t]{4}{*}{$\mathrm{DMC}^{(2)}$} & $\mathrm{Hm}$ & $67,80 \mathrm{~b}$ & $73,90 \mathrm{a}$ & $-0,31$ * \\
\hline & $\mathrm{Gt}$ & $57,50 \mathrm{~b}$ & $67,60 \mathrm{a}$ & $-2,35$ * \\
\hline & $\mathrm{Ct}$ & $35,00 \mathrm{a}$ & 33,81 a & $0,59 \mathrm{~ns}$ \\
\hline & $\mathrm{Gb}$ & $88,50 \mathrm{a}$ & $92,30 \mathrm{a}$ & $-1,80 \mathrm{~ns}$ \\
\hline \multirow{2}{*}{\multicolumn{2}{|c|}{$\begin{array}{l}\mathrm{Gt} /(\mathrm{Gt}+\mathrm{Hm}) \\
\mathrm{Ct} /(\mathrm{Ct}+\mathrm{Gb})\end{array}$}} & $0,126 \mathrm{a}$ & $0,097 \mathrm{~b}$ & 2,69 * \\
\hline & & $0,38 \mathrm{a}$ & $0,37 \mathrm{a}$ & $0,77 \mathrm{~ns}$ \\
\hline
\end{tabular}

(1) Largura à meia altura $\left({ }^{\circ} 2 \Theta\right)$. ${ }^{(2)}$ Diâmetro médio do cristal $(\mathrm{nm})$, ${ }^{*}$ significativo a $5 \%$ e ${ }^{\text {ns }}$ não-significativo pelo teste $t$-Student. Hm: hematita, Gt: goethita, Ct: caulinita, Gb: gibbsita. 
Quadro 3. Avaliação geoestatística dos atributos cristalográficos da hematita (Hm), goethita (Gt), caulinita (Ct) e gibbsita (Gb) e razão $\mathrm{Gt} /(\mathrm{Gt}+\mathrm{Hm})$ e $\mathrm{Ct} /(\mathrm{Ct}+\mathrm{Gb})$ de 119 amostras coletadas na profundidade de 0,2$0,4 \mathrm{~m}$

\begin{tabular}{|c|c|c|c|c|c|c|c|}
\hline & & Modelo & $\mathrm{C}_{0}{ }^{(1)}$ & $\mathrm{C}_{0}+\mathrm{C}_{1}{ }^{(2)}$ & $\mathbf{a}^{(3)}$ & $\mathrm{C}_{0} /\left(\mathrm{C}_{0}+\mathrm{C}_{1}\right)^{(4)}$ & $\mathrm{R}^{2^{(5)}}$ \\
\hline & & & & & & $\%$ & \\
\hline \multirow[t]{4}{*}{$\mathrm{LMA}^{(6)}$} & $\mathrm{Hm}$ & Exponencial & 0,00055 & 0,00103 & 56,1 & 5,31 & 0,744 \\
\hline & $\mathrm{Gt}$ & Exponencial & 0,01126 & 0,02322 & 13,5 & 47,82 & 0,313 \\
\hline & $\mathrm{Ct}$ & Exponencial & 0,00060 & 0,00323 & 18,0 & 18,57 & 0,332 \\
\hline & $\mathrm{Gb}$ & Exponencial & 0,00006 & 0,00013 & 18,1 & 46,15 & 0,467 \\
\hline \multirow[t]{4}{*}{$\mathrm{DMC}^{(7)}$} & $\mathrm{Hm}$ & Esférico & 124,12678 & $198,803 \quad 41$ & 37,2 & 62,44 & 0,543 \\
\hline & $\mathrm{Gt}$ & Esférico & 320,74602 & 528,60537 & 19,8 & 60,67 & 0,462 \\
\hline & $\mathrm{Ct}$ & Exponencial & 15,55074 & 99,47150 & 16,2 & 15,63 & 0,664 \\
\hline & $\mathrm{Gb}$ & Exponencial & 53,79320 & 126,21596 & 20,1 & 42,62 & 0,598 \\
\hline \multirow{2}{*}{\multicolumn{2}{|c|}{$\begin{array}{l}\mathrm{Gt} /(\mathrm{Gt}+\mathrm{Hm}) \\
\mathrm{Ct} /(\mathrm{Ct}+\mathrm{Hm})\end{array}$}} & Exponencial & 0,00209 & 0,00342 & 24,4 & 61,20 & 0,294 \\
\hline & & Exponencial & 0,00183 & 0,00311 & 26,6 & 58,84 & 0,655 \\
\hline
\end{tabular}

(1) Efeito pepita. ${ }^{(2)}$ Patamar. ${ }^{(3)}$ Alcance $(\mathrm{m}) .{ }^{(4)}$ Grau de dependência espacial. ${ }^{(5)}$ Coeficiente de determinação. ${ }^{(6)}$ Largura à meia altura $\left({ }^{\circ} 2 \theta\right) .{ }^{(7)}$ Diâmetro médio do cristal (nm).

(a) LMA da $\mathrm{Hm}\left({ }^{\circ} 2 \theta\right)$

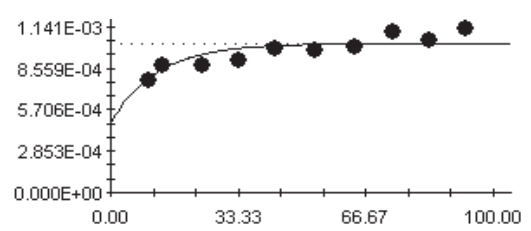

(c) LMA da Ct $\left({ }^{\circ} 2 \theta\right)$

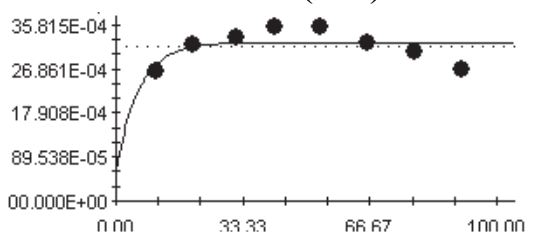

(e) DMC da Hm (nm)

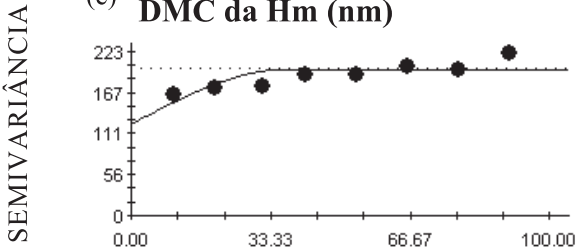

(g) DMC da Ct (nm)

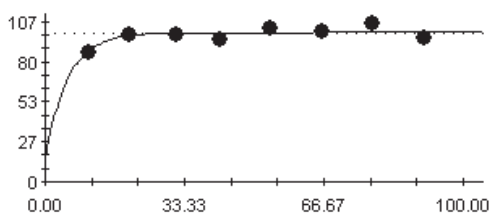

(i) Razão Gt/(Gt+Hm)

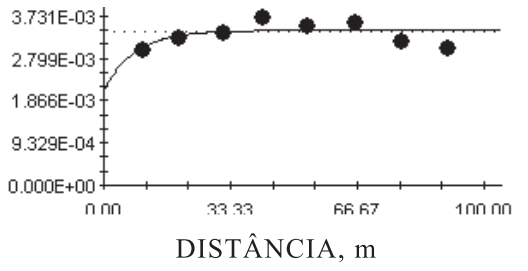

(b) LMA da Gt $\left({ }^{\circ}\right.$ 2 $\left.\theta\right)$

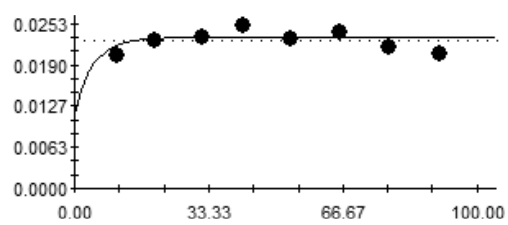

(d) LMA da Gb $\left({ }^{\circ} 2 \theta\right)$

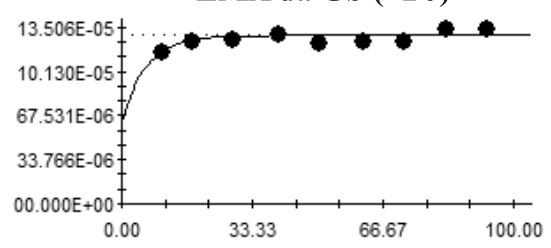

(f)

DMC da Gt (nm)

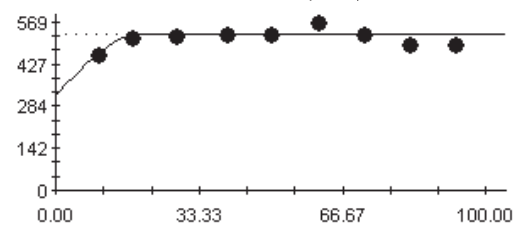

(h) DMC da Gb (nm)

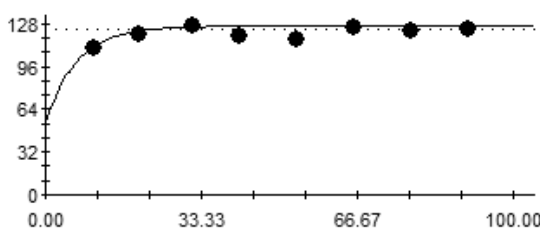

(j) Razão Ct $/(\mathbf{C} \mathbf{t}+\mathbf{G b})$

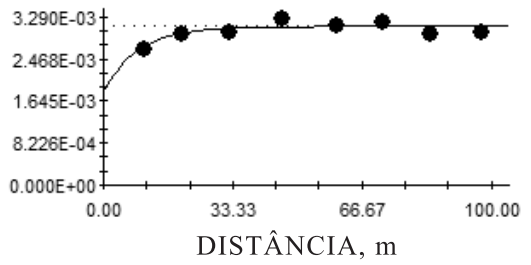

Figura 2. Semivariogramas dos atributos: largura à meia altura (LMA), diâmetro médio do cristal (DMC) dos minerais hematita $(\mathrm{Hm})$, goethita $(\mathrm{Gt})$, caulinita $(\mathrm{Ct})$ e gibbsita $(\mathrm{Gb})$ e razões $\mathrm{Gt} /(\mathrm{Gt}+\mathrm{Hm})$ e $\mathrm{Ct} /(\mathrm{Ct}+\mathrm{Gb})$. 
dependência espacial moderada $\left[\left(\mathrm{C}_{0} /\left(\mathrm{C}_{0}+\mathrm{C}_{1}\right)\right.\right.$ entre 25 e $75 \%$ ], segundo classificação proposta por Cambardella et al. (1994). Segundo esses autores, a dependência espacial forte das propriedades do solo pode ser controlada por variações intrínsecas nas características do solo, como textura e mineralogia.

Os atributos estudados apresentaram diferentes valores de alcance, e estes foram superiores ao valor de espaçamento utilizado na malha de amostragem, indicando que as amostras estão espacialmente relacionadas, o que permite que se façam interpolações (Vieira, 2000). Carvalho et al. (2002) afirmam que amostras devem ser coletadas a uma distância equivalente à metade do alcance. Ressalta-se que os maiores valores do alcance foram registrados para os atributos de LMA e DMC da hematita, e o menor valor, para os mesmos atributos relativos à goethita, indicando a menor variabilidade espacial da hematita. Isso está de acordo com Inda Júnior \& Kämpf (2005), que atribuem a este mineral populações mais homogêneas comparadas com as da goethita.

Os mapas do padrão espacial de todos os minerais, exceto da $\mathrm{Ct}$ (Figura 3), apresentaram menores valores relativos da LMA e maiores de DMC no
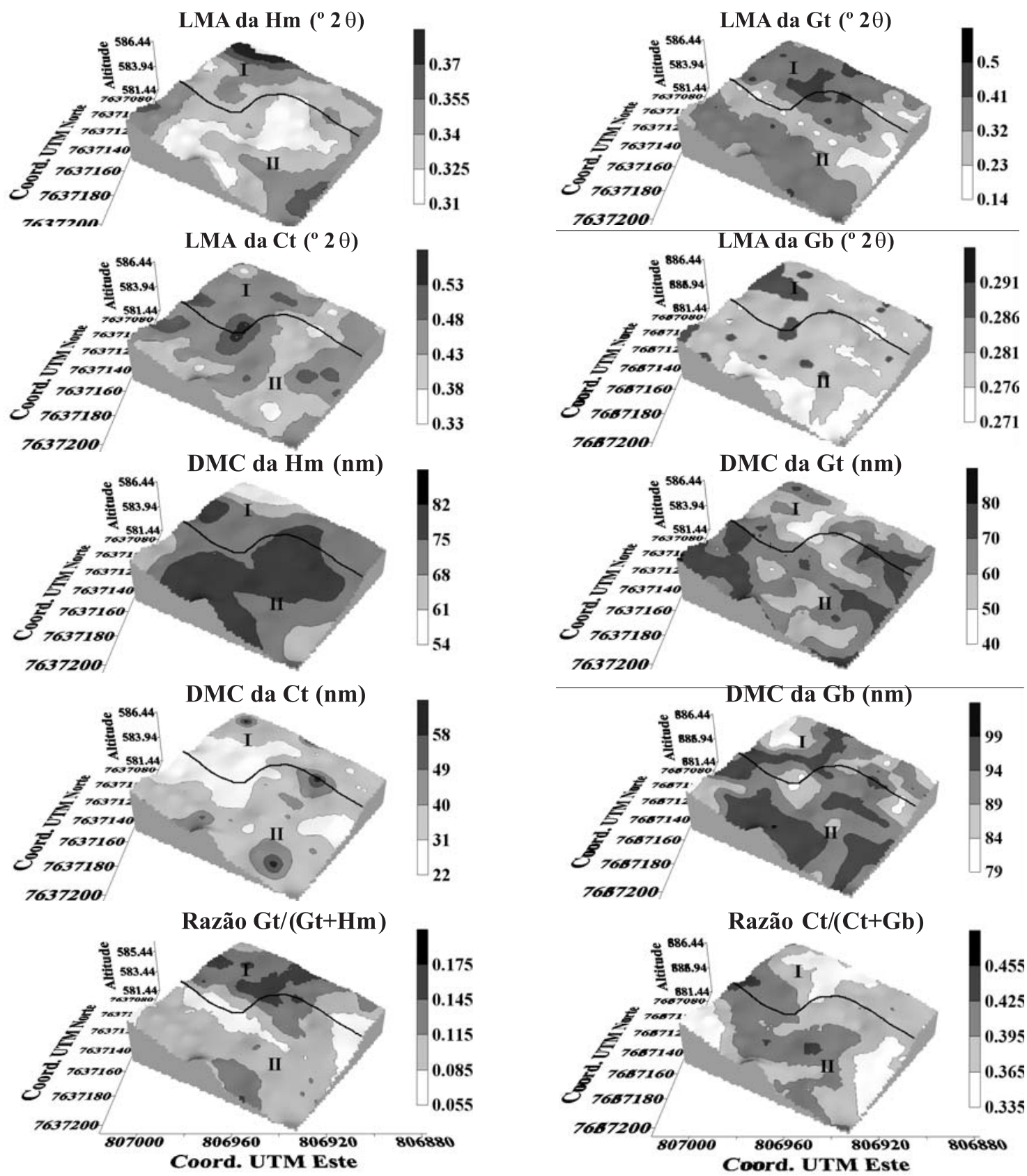

Figura 3. Mapas do padrão espacial dos atributos: largura à meia altura (LMA), diâmetro médio do cristal (DMC) dos minerais hematita $(\mathrm{Hm})$, goethita $(\mathrm{Gt})$, caulinita $(\mathrm{Ct})$ e gibbsita $(\mathrm{Gb})$ e razões $\mathrm{Gt} /(\mathrm{Gt}+\mathrm{Hm})$ e $\mathrm{Ct} /(\mathrm{Ct}+\mathrm{Gb})$. Coord.= coordenadas. 
compartimento II. Essa distribuição corrobora a diferença significativa para os valores dos atributos da Hm e Gt entre os compartimentos (Quadro 2), o que está de acordo com a maior dependência pedoambiental desses minerais (Kämpf \& Curi, 2000). A ocorrência de minerais com maior grau de cristalinidade no compartimento II indica que este é o ambiente preferencial de formação desses minerais.

Esses resultados estão de acordo com Curi \& Franzmeier (1984), Inda Júnior \& Kämpf (2005), quando afirmam que goethitas de maior grau de cristalinidade ocorrem em pedoambiente de maior relação $\mathrm{Gb} /(\mathrm{Gb}+\mathrm{Ct})$. A relação $\mathrm{Gt} /(\mathrm{Gt}+\mathrm{Hm})$ obteve os maiores valores no compartimento I. Segundo Daniels \& Hammer (1992), técnicas de amostragens de solo comumente usadas em trabalhos de geoestatística só auxiliam na transferência de informações, de uma área para outra, quando consideram a geomorfologia e a estratigrafia do terreno. Para esses autores, a idéia de que a variabilidade dos atributos do solo ocorre ao acaso é falsa, pois a maior parte dessa variabilidade é seqüencial e pode ser prevista quando se faz uso do modelo de paisagem. Essas predições são muito úteis, principalmente para execução de levantamentos pedológicos detalhados.

Os mapas do padrão espacial dos atributos mineralógicos dos minerais demonstram uma separação clara dos diversos ambientes pedogenéticos (Cunha, 2000), os quais coincidem com o modelo de curvaturas de Troeh (1965). Paz \& Taboado (1996) afirmaram que a utilização de altitude, declividade e formas da paisagem do solo para delinear zonas de manejo e estudo da variabilidade espacial dos atributos em diferentes tipos de solos é de extrema importância.

Neste estudo, observa-se que as formas do relevo se relacionaram com a formação e a cristalinidade dos minerais da fração argila, e as relações entre os atributos mineralógicos podem ser entendidas por meio do padrão de distribuição destes na paisagem. O conhecimento do padrão espacial dos atributos mineralógicos revela o potencial para o entendimento e a identificação dos ambientes de formação dos minerais da fração argila.

\section{CONCLUSÕES}

1. Todos os atributos cristalográficos estudados apresentaram dependência espacial.

2. A gibbsita apresentou o maior diâmetro médio do cristal entre os minerais da fração argila estudados, e a goethita foi o mineral que apresentou a maior variação do diâmetro médio do cristal e da largura à meia altura.

3. Os maiores valores do diâmetro médio do cristal da goethita, hematita e gibbsita foram encontrados no compartimento II, caracterizado por uma área côncavo-convexa.

\section{AGRADECIMENTOS}

À Usina São Martinho, pela concessão da área de estudo.

À Fundação de Amparo à Pesquisa do Estado de São Paulo (FAPESP), pela concessão de bolsa à primeira e quarta autoras.

\section{LITERATURA CITADA}

CAMBARDELLA, C.A.; MOORMAN, T.B.; NOVAK, J.M.; PARKIN, T.B.; KARLEN, D.L.; TURCO, R.F. \& KONOPKA, A.E. Field-scale variability of soil properties in Central Iowa Soil. Soil Sci. Soc. Am. J., 58:1501-1508, 1994.

CARVALHO, J.R.P.; SILVEIRA, P.M. \& VIEIRA, S.R. Geoestatística na determinação da variabilidade espacial de características químicas do solo sob diferentes preparos. Pesq. Agropec. Bras., 37:1151-1159, 2002.

CRESSIE, N. Statistics for spatial data. New York, John Wiley, 1991. 900p.

CUNHA, P. Superfícies geomórficas e variabilidade de Latossolos em uma vertente sobre arenito-basalto em Jaboticabal (SP). Jaboticabal, Universidade Estadual Paulista, 2000. 149p. (Tese de Doutorado)

CURI, N. \& FRANZMEIER, D.P. Toposequence of Oxisols from the central plateau of Brazil. Soil Sci. Soc. Am. J., 48:341-346, 1984

DANIELS, R.B. \& HAMMER, R.D. Soil geomorphology. New York, John Wiley, 1992. 236p.

DICK, D.P. Caracterização de óxidos de ferro e adsorção de fósforo na fração argila de horizontes B latossólicos. Porto Alegre, Universidade Federal do Rio Grande do Sul, 1986.196p. (Tese de Mestrado)

EMPRESA BRASILEIRA DE PESQUISA AGROPECUÁRIA EMBRAPA. Centro Nacional de Pesquisa de Solos. Sistema brasileiro de classificação de solos. 2.ed. Rio de Janeiro, 2006. p.306.

FERREIRA, M.M.; FERNANDES, B. \& CURI, N. Influência da mineralogia da fração argila nas propriedades físicas de Latossolos da região Sudeste do Brasil. R. Bras. Ci. Solo, 23:515-524, 1999a.

FERREIRA, M.M.; FERNANDES, B. \& CURI, N. Mineralogia da fração argila e estrutura de Latossolos da região Sudeste do Brasil. R. Bras. Ci. Solo, 23:507-514, 1999 b.

FITZPATRICK, R.W. \& SCHWERTMANN, U. Al-Substituted goethite na indicator of pedogenic and other weathering environments in South Africa. Geoderma, 27:335347,1982 .

GHIDIN, A.A.; MELO, V.F.; LIMA, V.C. \& LIMA, J.M.J.C. Toposseqüências de Latossolos originados de rochas basálticas no Paraná. I - Mineralogia da fração argila. R. Bras. Ci. Solo, 30:293-306, 2006. 
HAMLETT, J.M.; HORTON, R. \& CRESSIE, N. Resistant and exploratory techniques for use in semivariogram analyses. Soil Sci. Soc. Am. J., 50:868-875, 1986.

HAMMER, R.D.; YOUNG, N.C.; WOLENHAUPT, T.L.; BARNEY, T.L. \& HAITHCOATE, T.W. Slope class maps form soil survey and digital elevation models. Soil Sci. Soc. Am. J., 59:509-519, 1995.

INDA JÚNIOR, A.V. \& KÄMPF, N. Variabilidade de goethita e hematita via dissolução redutiva em solos de região tropical e subtropical. R. Bras. Ci. Solo, 29:851-866, 2005.

KÄMPF, N. \& CURI, N. Óxidos de ferro: Indicadores de atributos e ambientes pedogenênicos e geoquímicos. In: NOVAIS, R.F.; ALVAREZ V., V.H. \& SCHAEFER, C.E.G.R., eds. Tópicos em ciência do solo. Viçosa, MG, Sociedade Brasileira de Ciência do Solo, 2000. p.107-138.

KÄMPF, N. \& SCHWERTMANN, U. Avaliação da estimativa de substituição de $\mathrm{Fe}$ por $\mathrm{Al}$ em hematitas de solos. R. Bras. Ci. Solo, 22:209-213,1998.

KÄMPF, N. \& SCHWERTMANN, U. Goethite and hematite in a climosequence in Southern Brazil and their application in classification of kaolinitic Soils. Geoderma, 29:27-39, 1982.

KLINGEBIEL, A.A.; HORVATH, E.H.; MOORE, D.G. \& REYBOLD, W.U. Use of slope, aspect, and elevation maps derived from digital elevation model data in making soil surveys. In: REYBOLD, W.U. \& PETERSEN, G.W. Soil survey techniques. Madison, Soil Science Society of America, 1987. p.77-90. (SSSA Special Publication, 20)

McBRATNEY, A.B. \& WEBSTER, R. Choosing functions for semivariograms of soil properties and fitting them to sample estimates. J. Soil Sci., 37:617-637, 1986.

MEHRA, O.P. \& JACKSON, M.L. Iron oxide removal from soils and clay by a dithionite-citrate system bulfered with sodium bicarbonate. Clays Clay Miner., 7:317-327, 1960.

MELO, V.F.; FONTES, M.P.F.; NOVAIS, R.F.; SINGH, B. \& SCHAEFER, C.E.G.R. Características dos óxidos de ferro e de alumínio de diferentes classes de solo. R. Bras. Ci. Solo, 25:19-32, 2001.

MINITAB Release. Making data analysis easler: version 13.1, 2000.

MOTTA, P.E.F. \& KÄMPF, N. Iron oxide properties as support to soil morphological features for prediction of moisture regimes in Oxisols of Central Brazil. Z. Pflanzenernähr Bodenk., 155:385-390, 1992.

MUGGLER, C.C. Polygenetic oxisols on tertiary surfaces, Minas Gerais, Brazil. Wageningen, Wageningen Agricultural University, 1998. 186p. (Tese de Doutorado)

NORRISH, K. \& TAYLOR, R.M. The isomorphous replacement of iron by aluminium in soil goethites. J. Soil Sci., 12:294306,1961 .

PEDROTTI, A.; FERREIRA, M.M.; CURI, N.; SILVA, M.L.N.; LIMA, J.M. \& CARVALHO, R. Relação entre atributos físicos , mineralogia da fração argila e formas de alumínio no solo. R. Bras. Ci. Solo, 27:1-9, 2003.
PAZ, A.G. \& TABOADO, M.T. Medida del microrrelieve del suelo y estimación de la retención hídrica en depresiones de la superficie. J. Soc. Esp. Geomorf., 34:829-841, 1996.

ROBERTSON, G.P. $\mathrm{GS}^{+}$: Geostatistics for the environmental sciences - $\mathrm{GS}^{+}$user's guide. Plainwell, Gamma Design Soffware, 1998. 152p.

SCHULZE, D.G. The influence of aluminium on iron oxides VIII. Unit-cel dimension of Al-substituted of goethites and estimation of $\mathrm{Al}$ from them. Clays Clay Miner., 32:36$44,1984$.

SCHWERTMANN, U. \& CARLSON, L. Aluminium influence on iron oxides: XVII. Unit-cell parameters and aluminium substitution of natural goethites. Soil Sci. Soc. Am. J., 58:256-61, 1994.

SCHWERTMANN, U. \& KÄMPF, N. Properties of goethite and hematite in kaolinitic soils of Southern and Central Brazil. Soil Sci., 139:344-50, 1985.

SCHWERTMANN, U. \& TAYLOR, R.M. Iron oxides. In: DIXON, J.B. \& WEED, S.B., eds. Minerals in soil environments. 2.ed. Madison, Soil Science Society of America, 1989. p.379-438. (Book Series, 1)

SOUZA, Z.M.; SILVA, M.L.S.; GUIMARÃES, G.L.; CAMPOS, D.T.S.; CARVALHO, M.P. \& PEREIRA, G.T. Variabilidade espacial de atributos físicos em um Latossolo Vermelho distrófico sob semeadura direta em Selvíria (MS). R. Bras. Ci. Solo, 25:699-707, 2001.

STRAUSS, R.; BRÜMMER, G.W. \& BARROW, N.J. Effects of crystallinity of goethite: II. Rates of sorption and desorption of phosphate. Eur. J. Soil Sci., 48:101-114, 1997.

SURFER. Surfer 7.0. Contouring and 3D surface mapping for scientist's engineers: User's guide. New York, Golden software, 1999. 619p.

TRANGMAR, B.B.; YOST, R.S. \& UEHARA, G. Application of geostatistics to spatial studies of soil properties. Adv. Agron., 38:54-94, 1985.

TROEH, F.R. Landform equations fitted to contour maps. Am. J. Sci., 263:616-627, 1965.

VIEIRA, S.R. Geoestatística em estudos de variabilidade espacial do solo. In: NOVAIS, R.F.; ALVAREZ, V., V.H. \& SCHAEFER, G.R., eds. Tópicos em ciência do solo. Viçosa, MG, Sociedade Brasileira de Ciência do Solo, 2000. v. 1. p.1-54.

VIEIRA, S.R. Uso da geoestatística em estudos de variabilidade espacial. In: CURSO de atualização em conservação do solo. Campinas, IAC, 1995. 61p.

VIEIRA, S.R. Variabilidade espacial de argila, silte e atributos químicos em uma parcela experimental de um Latossolo Roxo de Campinas (SP). Bragantia, 56:181-90, 1997.

VIEIRA, S.R.; MILLETE, J.; TOPP, G.C. \& REYNOLDS, W.D. Handbook for geostatistical analysis of variability in soil and climate data. In: ALVAREZ V., V.H.; SCHAEFER, C.E.G.R.; BARROS, N.F.; MELLO, J.W.V. \& COSTA, L.M., eds. Tópicos em ciência do solo. Viçosa, MG, Sociedade Brasileira de Ciência do Solo, 2002. v.2. p.1-45. 